\title{
An evolving spatio-temporal approach for gender and age group classification with Spiking Neural Networks
}

Alvi, F., Pears, R., \& Kasabov, N. (2018). An evolving spatio-temporal approach for gender and age group classification with Spiking Neural Networks. Evolving Systems, 9((2018)), 145-156.

https://doi.org/10.1007/s12530-017-9175-y

Link to publication record in Ulster University Research Portal

Published in:

Evolving Systems

Publication Status:

Published (in print/issue): 30/06/2018

DOI:

10.1007/s12530-017-9175-y

\section{Document Version}

Author Accepted version

\section{General rights}

Copyright for the publications made accessible via Ulster University's Research Portal is retained by the author(s) and / or other copyright owners and it is a condition of accessing these publications that users recognise and abide by the legal requirements associated with these rights.

\section{Take down policy}

The Research Portal is Ulster University's institutional repository that provides access to Ulster's research outputs. Every effort has been made to ensure that content in the Research Portal does not infringe any person's rights, or applicable UK laws. If you discover content in the Research Portal that you believe breaches copyright or violates any law, please contact pure-support@ulster.ac.uk. 


\section{An evolving spatio-temporal approach for gender and age group classification with Spiking Neural Networks}

Fahad Bashir Alvi, Russel Pears, Nikola Kasabov

Received: date / Accepted: date

\begin{abstract}
This research study proposes a novel method of inter-related problems in face recognition using the NeuCube neuromorphic computational platform. We investigated age classification and gender recognition. The well-known FG-NET and MORPH Album 2 image gallery were used and anthropometric features were extracted from landmark points on the face. The landmarks were pre-processed with the procrustes algorithm before feature extraction was performed. The Weka machine learning workbench was used to compare the performance of traditional techniques such as the K nearest neighbor (Knn) and Multi-LayerPerceptron (MLP) with NeuCube. Our empirical results show that NeuCube performed consistently better across both problem types that we investigated.
\end{abstract}

Keywords Anthropometric Model, Age Group Classification, Gender Classification, Spiking Neural Networks

\section{Introduction}

Aging is a slow process and its effects are visible only after a few years. But in spite of being slow, it remains

(凶) Fahad Bashir Alvi

Knowledge Engineering and Discovery Research Institute

Auckland University of Technology, Auckland, New Zealand

E-mail: fahadalvi@hotmail.com, falvi@aut.ac.nz

- Russel Pears

School of Computer and Mathematical Sciences

Auckland University of Technology, Auckland, New Zealand

E-mail: rpears@aut.ac.nz

- Nikola Kasabov

Knowledge Engineering and Discovery Research Institute

Auckland University of Technology, Auckland, New Zealand

E-mail: nkasabov@aut.ac.nz a spatio-temporal phenomenon. The facial features of a person can be considered as a subspace and the aging over the years of this subspace is in turn a temporal process. It would be very useful to incorporate the temporal as well as spatial patterns in aging data as important components in classification. The raw data which has been used in this study is from (FG-NET) and MORPH image galleries. Age group classification and gender recognition have important applications for business managers and law enforcement agencies. In Human Computer Interaction (HCI) gender recognition can be used to make it more amenable to both genders. For example, it enables a computer to address a user by their correct title, Mr or Mrs, as the case may be. Automatic gender recognition facilitates better interaction with humans as well as saves keystrokes in filling up forms. In Surveillance systems, a gender specific physical locality can be concentrated upon, thereby reducing the area under observation and making the whole system more efficient. It could also assign higher threat levels to a specified gender location. In content based systems, indexing and searching can be greatly facilitated. For example, today a plethora of digital videos and photographs are produced. Their gender based indexing and searching can be easily carried out. It will also reduce the search effort by limiting it to a particular gender, thereby making it more efficient. The same applies to Biometrics. The automatic collection of demographic data for statistical purposes will be facilitated if the gender can be automatically recognised.

Age group classification(Kwon and da Vitoria Lobo 1999; Horng et al 2001; Lanitis et al 2004; Gunay and Nabiyev 2008; Wang et al 2009, 2011; Thukral et al 2012; Liu et al 2015) and gender recognition(Guo and Mu 2011; Levi and Hassner 2015) is also an area of research that is receiving a lot of attention due to ad- 
vances in the field of biometrics (Guo and Mu 2011; Jain et al 2016; Liu et al 2015; Ramanathan and Chellappa 2006). There are a number of challenges in making an effective age group classification and gender recognition system, which include: pose, illumination, face expression, occlusions and aging effects. It is challenging to address all of them simultaneously, because if one error is removed then another crops up. We have adopted the approach of using Anthropometric features (Ramanathan and Chellappa 2006) which eliminates some of these problems. This model is based on geometrical ratios and distances of fixed number of fiducial landmarks, which in our case corresponds to 68 landmark points on the face. Since the Anthropometric model uses geometrical distances it is not affected by texture information, illumination and intensity levels. Problems caused by occlusions such as moustaches or spectacles are also eliminated as the distance between features will remain unchanged even if such occlusions occur. However, different poses could create problems(Alvi and Pears 2015a,b,c, 2016). We mitigate the effects of this problem by finding an average of all faces and then warping all images to that image with the help of the procrustes algorithm (Cootes et al 1999). This allow us to concentrate on effects due to aging. Aging not only changes texture, but also alters geometrical ratios and distance between landmark points. However, such changes over time follow a definite pattern. Thus, our main research hypothesis is that our anthropometric model would provide a realistic basis for studying the effects of aging due to change in geometry of the face.

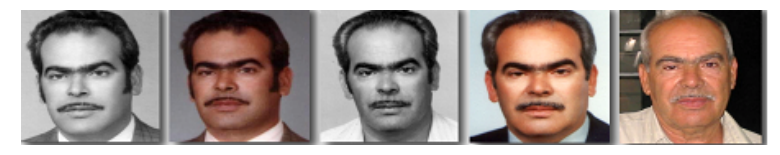

Fig. 1 Same Individual at different ages (FG-NET 2002) .

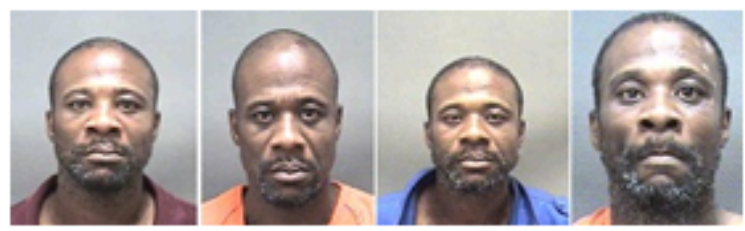

Fig. 2 Same Individual at different ages from (Ricanek Jr and Tesafaye 2006).

This Research focuses on designing a novel framework for age group classification and gender recognition. We use an Anthropometric model for feature ex- traction and extract seven different indexes. These indexes consist of different distances and geometrical ratios of face features. After extracting features we convert the data into a discrete spike train. We use the AER encoding method to discretize the continuous signal. This encoding method was applied successfully for the artificial retina sensor (Hechenbichler and Schliep 2004). We initialize the NeuCube by using the Small World rule (Hechenbichler and Schliep 2004). At unsupervised training stage we used the STDP rule (Hechenbichler and Schliep 2004). The dynamic evolving Spike Neural Networks (deSNN) is used here as an output classifier. Our choice of the NeuCube platform was influenced by its outstanding success that it has achieved on spatio-temporal classification problems in many diverse (Kasabov et al 2016; Dhoble et al 2012; Kasabov et al 2013; Kasabov 2014; Sengupta et al 2015; Kasabov 2007). All such studies employing NeuCube have been on data that exhibit a relatively fast pace of temporal changes. However in the aging domain that this research is based on, the speed of changes are much smaller, on the order of months to years rather than hours to seconds, and hence it will be interesting to investigate whether the success of NeuCube can be replicated in such a slow-changing temporal environment. Our empirical results indicate that NeuCube captures temporal patterns well in this domain as well, as its classification accuracy was significantly better than with classical methods such as the K Nearest Neighbor(KNN), Multi Layer Perceptron (MLP) and Naive Bayes classifiers.

\section{Literature Review}

(Kwon and da Vitoria Lobo 1999) proposed a classification approach based on age that identifies the age group of given face images. It is based on the anthropometry of the face and the density of its face wrinkles. They considered three age groups in their study. These groups were infants, young adults and senior adults. They also observed that the lower and upper halves of faces grow at different rates during the early formative years.

(Lanitis et al 1999) also used Active Appearance Models (AAM). Lanitis et al used a statistical face model for studying the age estimation problem. AAM parameters were extracted from facial images which were marked with 68 points. A Genetic Algorithm was then applied to build and optimize an aging function.

(Geng et al 2007) worked on the assumption that similar faces age in similar ways across a population. He introduced the method of Aging pattern Subspace 
(AGES) for modelling aging across time. Geng et al constructed a representative subspace by utilizing a sequence of images of a given individuals face sorted in chronological order. Thereafter, minimum reconstruction error was achieved by projecting the unseen face image into its proper aging subspace, thus making it possible to determine the age of the image from its aging pattern.

(Yan et al 2008) introduced a method which developed the concept of coordinate patches and Gaussian Mixture Models (GMMs) which were used to estimate facial ages. In their method, the face image of an individual is encoded as a group of overlapped spatially fexible patches (SFPs). Local features are then extracted by a $2 \mathrm{D}$ discrete cosine transform (DCT). The patches are used for integration of local features and coordinate information. The extracted SFPs are then modelled with Gaussian Mixture Models (GMMs). The model produced was used to estimate age of a person by comparing the sum of likelihoods from total spatially fexible patches (SFPs) of an hypothetical age.

(Guo et al 2008) designed a localized regression classifier to learn the aging function. The concept of manifold learning was used for age estimation. (Fu and Huang 2008) also used manifold learning as well. A set of age separated images were taken and a low dimensional manifold was developed from it. Linear and quadratic regression functions were applied on the low dimensional feature vectors from the respective manifolds.

Face anthropometry is a science of measuring sizes and proportions on human faces. It is found that it can play an important role in developing facial aging models. These sizes and ratios provide a quantitative description of the craniofacial complex at different ages. This provides numerous options for learning based approaches. These approaches are developed to characterize facial aging. Face anthropometric studies take measurements between key landmarks on human faces at different ages. These ratios have played a critical role in surgical procedures employed on the faces of growing children (Farkas and Munro 1987).

(Farkas 1994) provides a comprehensive overview of face anthropometry and also describes its many significant applications. He has taken 57 selected landmarks on human face that are spread acroos 66 regions in the craniofacial complex (head, face, orbits, nose, lips and mouth, ear). The facial measurements are of of three kinds: (i) projective measurements (shortest distance between two land- marks)(ii) tangential measurements (distance between two landmarks measured along the skin surface) (iii) angular measurements. Figure 3 illustrates the kind of data that is collected in face an- thropometric studies and further illustrates the different fiducial features across which such data is collected. Since age-based anthropometric data was not available, they prepared it by extracting facial features from passport database. The data was grouped into 5 age groups, namely 21-30 yrs, 31-40 yrs, 41-50 yrs, 51-60 yrs and 61- 70 yrs. Such data was found to be effective in characterizing facial growth based on age, gender, ethnicity etc. It could also characterise instances when individuals gain or lose weight. Thus it describes the process of transforming facial appearances with age progression (Farkas 1994).

(Biswas et al 2008) proposed that there is coherency in facial feature drifts across ages. The degree of drift was used as a measure to perform face verification across ages. The assumption was that facial feature drifts observed in images that are age separated follows a definite coherent drift pattern. It was also observed that the same might not be true for age separated face images of two different individuals. Biswas et al suggested that since fiducially extracted features on the outer contour tends to be very sensitive to head pose variations, a suitable pose correction mechanism had to be put in place.

Ramanathan and Chellappa (Ramanathan and Chellappa 2006) used eight ratios of distance measures to model age progression in young individuals, ranging in age from 0 to 18 years. These ratios were used to estimate an individual's appearance across time and to perform face recognition across age progression. They experimented with a database of 233 images of 109 individuals, partially collected from the FG-NET aging database and partially from a private gallery of images. Improvements in rank 1 accuracy of 8 percent (without age prediction) to 15 percent (with age transformation) was reported.

(Liu et al 2015) proposed a multistage learning system calling it Grouping Estimation Fusion (GEF). It addresses three research issues: age grouping, age estimation and decision fusion for finalizing age estimation. Global and local features are extracted and different groupings are created. A pipelined approach is used, whereby the output of each stage is fed as input to the next stage. The author has proposed six fusion schemes (i.e., intra-system fusion, inter- system fusion, intra-inter fusion, inter-intra fusion, maximum- diversity fusion, and composite fusion) are developed and compared. The performance of the GEF system is evaluated on FG-NET and MORPH databases. 


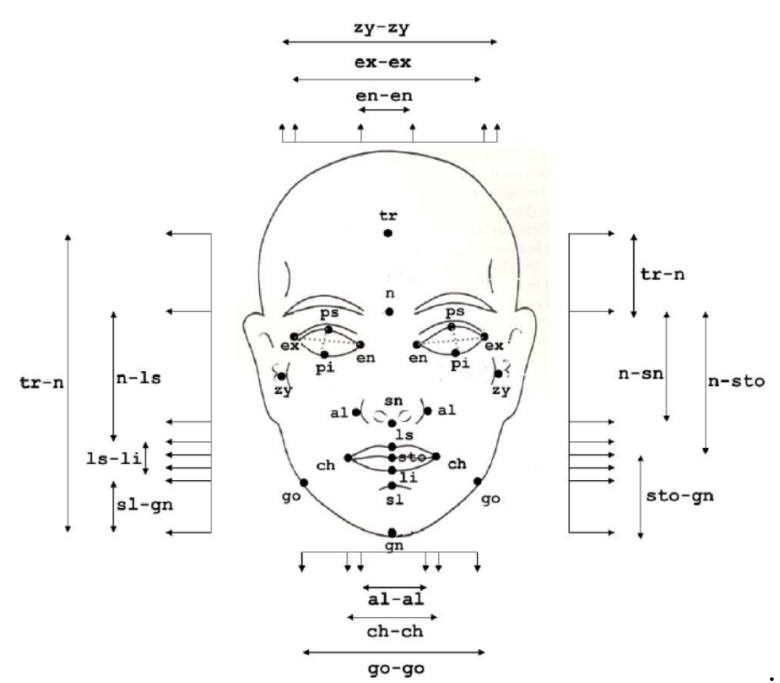

- Facial index $\left(\frac{n-g n}{z y-z y}\right)$

- Mandibular index $\left(\frac{s t o-g n}{g o-g o}\right)$

- Intercantal index $\left(\frac{e n-e n}{e x-e x}\right)$

- Orbital width index $\left(\frac{e x-e n}{e n-e n}\right)$

- Eye fissure index $\left(\frac{p s-p i}{e x-e n}\right)$

- Vermilion height index $\left(\frac{l s-s t o}{s t o-l i}\right)$

- Mouth Face width index $\left(\frac{c h-c h}{z y-z y}\right)$

Fig. 3 Anthropometric Model and Seven Features (Farkas 1994).

\section{Methodology}

An Anthropometric model was used in our study. A set of landmarks (a total of 68) was taken on each face, and distances between landmarks as well as ratios between distances were taken for constructing fiducial features. We used 7 such features on the face. The details of the model are given below. Since the images in the database are not of the same size, we applied procrustes to align them to a fixed size. This size was determined by averaging all the images in the database. The final feature determination was done after application of procrustes on landmark coordinates. These features were then fed into NeuCube. NeuCube, featuring a spiking neural network architecture that was successfully used in spatio-temporal modelling in brain data applications (Kasabov 2014; Kasabov et al 2014; Kasabov 2012; Kasabov et al 2015; Tu et al 2014). In contrast to brain data signals obtained through the use of devices such as EEG where time length between observations spans seconds, the corresponding time length in facial aging spans years, thus making it an interesting experiment to test the efficacy of NeuCube's spatio-temporal modelling capability in an environment of much coarser time granularity. A block diagram of NeuCube's architecture is shown in Fig. 4. NeuCube consists of three major components: an input encoding module, a threedimensional Spiking Neural Network Cube(SNNc) and an output dynamic evolving spike neural network (deSNN) classifier (Kasabov 2012). In the first stage we adjust the connection weights in spiking SNNc to learn spatio temporal patterns embedded in the data. In the second stage, supervised learning is carried out.

Overall, the modeling process consists of data encoding, SNNc initialization, unsupervised training of the SNNc and finally supervised training of the classifier. The experiments were performed with CPU of Intel Core i7-2640 M Processor 2.8GHz, Memory of 8 GB, Windows 7 Professional (64 bit) and MatLab (64 bit) R2013a.

\subsection{Data Encoding}

In the aging problem feature data is in the form of real values, and hence needs to be discertized into spike trains. The Address Event Representation (AER) encoding method is used for this purpose. This encoding method was applied successfully for the artificial retina sensor (Hechenbichler and Schliep 2004; Schliebs et al 2012). We obtain both positive and negative spike trains for encoding. Positive spikes accompany an increase in feature value, whereas a negative spike occurs when the feature value has decreased.

\subsection{NeuCube(ST) Initialization}

We initialize the SNNc by the following rule. Each neuron in the $\mathrm{SNNc}$ is connected to its nearby neurons which are within a distance $\mathrm{d}$, where d equals the longest distance between any pair of neurons in the SNNc multiplied by a parameter $\mathrm{r}$. The initial weights are set with $\mathrm{r}$ set at random in the range $[-1,1]$. We randomly select $80 \%$ of the connection weights to be positive and the remaining $20 \%$ to be negative in the cube initialization process. The actual proportion of positive to negative connections will change continuously due to weight propagation that occurs when data is processed by the cube.

\subsection{Training Stage I:}

This stage deals with Unsupervised Reservoir Training. It encodes hidden spatio-temporal relationships from 


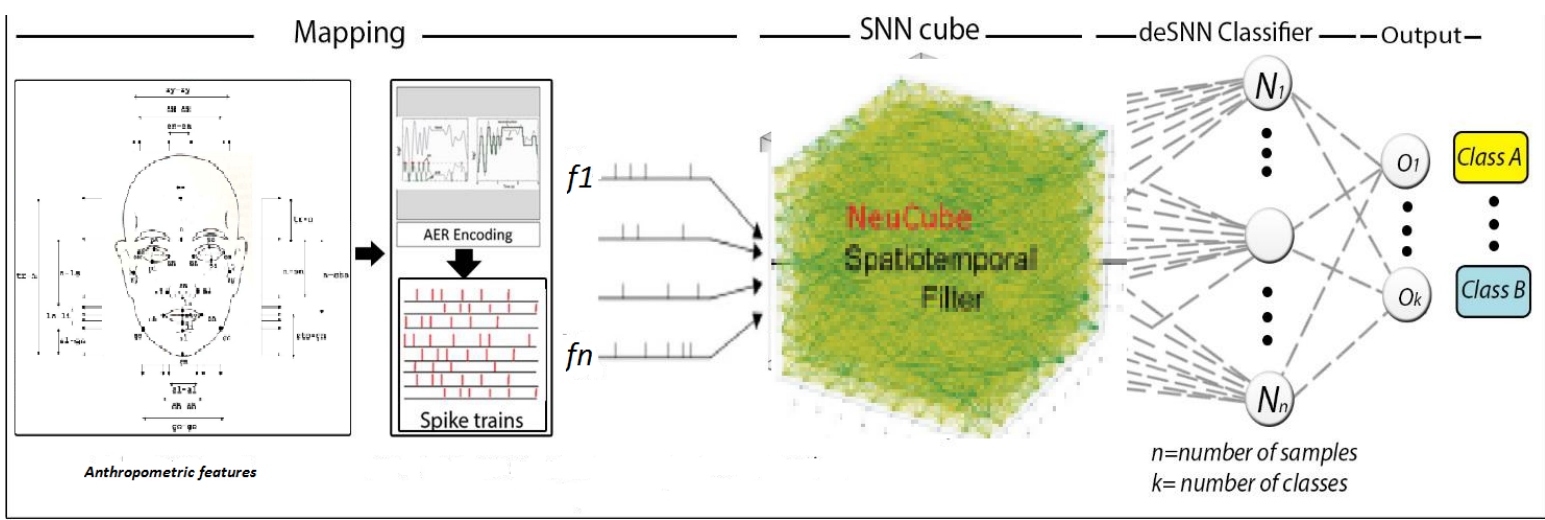

Fig. 4 Schematic representation of the NeuCube-based methodology for mapping, learning, visualisation and classification of FG-NET DATA

the input data into neuronal connection weights. According to Hebbian learning rule, if the interaction between two neurons persists, then the connection between them will be strengthened. We train the SNNc using the STDP learning rule (Song et al 2000): if neuron $\mathrm{j}$ fires before neuron $\mathrm{i}$, then the connection weight from neuron $\mathrm{j}$ to neuron $\mathrm{i}$ will increase, otherwise the connection from neuron i to neuron $\mathrm{j}$ will decrease. This ensures that the time signal inherent in the input spiking trains will be captured by the neuron firing state and manifest as asymmetrical connection weights in the SNNc.

In the $\mathrm{SNNc}$, when a neuron fires, it emits a spike and then its potential gradually reduces over time to reach a zero value. Each neuron connecting to this firing neuron will receive a spike. Because of the emitted spike the potential of each neighboring neuron will increase in proportion to its connection weight to the current firing neuron. The potential of each neuron has a small, constant rate of leakage over time until it reduces to 0 . After learning, the connection weights in the SNNc encode temporal relationships from the input spatiotemporal data ( $\mathrm{Tu}$ et al 2014).

\subsection{Training Stage II:}

In this stage, supervised classifier training is carried out. The deSNN (Dhoble et al 2012; Kasabov et al 2013; Kasabov 2014) is used here as an output classifier, because deSNN is computationally efficient and emphasizes the importance of the first spike, which has also been observed in biological systems.

Once the NeuCube(ST) Kasabov (2014) is trained, all connection weights in the $\mathrm{SNNc}$ and in the output classification layer are established. For a given new sample without any class label information, the trained NeuCube can be used to predict its class label. For the
deSNN classifier, there are two algorithms that can be used to determine the class label of the new sample (Dhoble et al 2012; Kasabov et al 2013; Schliebs and Kasabov 2013; Kasabov 2014). The deSNN classifier uses one of two algorithms to determine the class label of the new sample. One is (Dhoble et al 2012; Kasabov et al 2013; Kasabov 2014) and other is deSNNm (Dhoble et al 2012; Kasabov et al 2013; Kasabov 2014) . We have used the deSNNs algorithm in this work.

\section{Empirical Results of Age group Classification}

Experiments were performed on the publicly available FG-NET (FG-NET 2002) and MORPH Album 2 (the largest publicly available face aging dataset) (Ricanek $\mathrm{Jr}$ and Tesafaye 2006), both of which are used for benchmarking new methods. The lack of a large face aging database until recently limited research on age group classification. There are two desired attributes of a face aging database: (i) large number of subjects, and (ii) large number of face images per subject captured at many different ages ( $\mathrm{Li}$ et al 2011). In addition, it is desired that these images should not have large variations in pose, expression, and illumination. The MORPH dataset has a large number of subjects while FGNET database has a large number of images. The MORPH dataset contains about 55,000 face images from 13,000 different people.

In all experiments, the size of the SNNc is 1000 neurons, a relatively simple $10 \times 10 \times 10$ cube. It is trained and tested using a leave one out method. Firstly we converted the data into discrete spike trains using the AER encoding method to discretize the continuous signal, following the example of the silicon retina (Hechenbichler and Schliep 2004). The deSNN classifier mentioned previously is used here as an output classifier because deSNN is computationally efficient and emphasizes the importance of the first spike which has been 
observed to be significant in biological vision systems. We conducted experiments to compare traditional modeling methods (Knn, Niave Bayes and MLP) and our pro-posed method for age group classification.

We designed three experiments for these baseline algorithms. Note that for these baseline algorithms, the time length of training samples and testing samples have to be the same as these methods cannot tolerate different lengths of feature vectors for training and testing. Table 3 shows clearly that the classification achieved with NeuCube significantly outperformed the other techniques. The results clearly indicate that NeuCube with its spatio temporal capability can capture the aging effects more effectively than classical classifiers that do not explicity take into account the time dimension in the data.

Since evolving system data machines (eSTDM) model relationships both between and within spatio-temporal data, even a small amount of input data will be able to trigger the spiking activities in $\mathrm{SNNc}$, giving rise to a more accurate pattern (class) recognition rate from image data.

In order to assess generality of our models on different types of databases, we used MORPH Album 2 (the largest publicly available face aging dataset) database. We annotated the fiducial landmarks with Stasm, a method based on Active shape Models (Milborrow and Nicolls 2014).

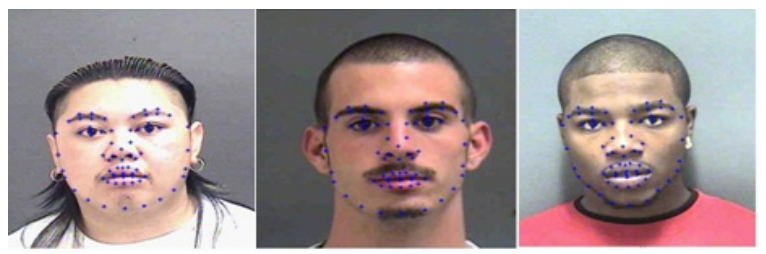

Fig. 5 Fiducial landmark detection results. Blue points represent the 68 landmark points images we take 7 features. Each sample provides a 5x7 matrix with each having 7 features. In all there are 90 samples. The leave-one-person-out testing strategy is used for the FG-NET database. For comparative purposes we used the same testing strategy as in (Liu et al 2015).

The data was then fed to NeuCube and Weka. The results of this experiment are given below.

Table 1 Age Group Classification using a NeuCube eSTDM in comparison to traditional classifiers: Knn, MLP, NB.

\begin{tabular}{llccl}
\hline Measure & NeuCube & MLP & knn & NaiveBayes \\
\hline \hline Accuracy(\%) & 98 & 80 & 91.1 & 66.7 \\
\hline
\end{tabular}

We benchmarked our NeuCube results against classical classifiers such as the $\mathrm{k}$ nearest neigbor, Multi Layer Perceptron and Naive Bayes.

Table 2 Age Group Classification using a NeuCube eSTDM for each age group separately

\begin{tabular}{llll}
\hline Age groups & $0-3$ & $4-15$ & $16-69$ \\
\hline \hline Accuracy(\%) & 100.00 & 96.00 & 98.00 \\
\hline
\end{tabular}

Table 3 Comparative results of age group classification with state-of-the-art age group classifiers

\begin{tabular}{l|l|}
\hline \hline Models & Classification(\%) \\
\hline \hline NeuCube & $98 \%$ \\
(Liu et al 2015) & $93.5 \%$ \\
(Sai et al 2015) & $90 \%$ \\
\hline \hline
\end{tabular}

Table 1 shows that NeuCube showed outperformed all of the general purpose classifiers, with the $\mathrm{k}$ nearest neighbor performing best amongst them.

Table 2 shows that Age Group Classification using a NeuCube eSTDM for each age group separately for FG-NET database.

Table 3 shows that the purpose-built age classifiers performed better than their general purpose counterparts. However, a substantial gap still exists between them and NeuCube. 


\subsection{Age Group Classification with MORPH}

We randomly selected a subset of about 21000 faces from MORPH database that contain black and white, Female and Male faces. Each sample provided a 5x7 matrix with each having 7 features. Our setting of the experiment is similar to that of (Liu et al 2015) and (Guo and $\mathrm{Mu}$ 2011). We divide data into three subsets, S1, S2 and S3. We used S1 for training and tested it with $\mathrm{W} / \mathrm{S} 1$. Then we used S2 for training and tested with W/S2. Finally, we averaged the two results to obtain an overall result.

Table 4 Results of Age Group Classification using a NeuCube eSTDM in comparison to traditional classifiers: SVM, MLP, NB.

\begin{tabular}{lllll}
\hline Measure & NeuCube & MLP & Knn & NaiveBayes \\
\hline \hline Accuracy(\%) & 95.00 & 82.30 & 89.03 & 67.60 \\
\hline
\end{tabular}

Table 5 Age Group Classification using a NeuCube eSTDM for each age group separately

\begin{tabular}{llll}
\hline Age groups & $15-29$ & $30-49$ & $50-77$ \\
\hline \hline Accuracy $\%)$ & 98.00 & 91.00 & 96.00 \\
\hline
\end{tabular}

Table 6 Comparative results of age group classification with Morph Album 2

\begin{tabular}{l|l}
\hline \hline Models & Classification(\%) \\
\hline \hline NeuCube & $95 \%$ \\
(Liu et al 2015) & $92.6 \%$ \\
(Sai et al 2015) & $71.2 \%$ \\
\hline \hline
\end{tabular}

Tables 4, 5 and 6 mirror the results for the FGNET database, once again underlining the superior performance of NeuCube.

Discussion In Figures 6, 7 and 8, blue lines represent positive connections (i.e. those with positive weights) while red lines represent negative connections (those with negative weights). The colour of a neuron signifies its connectivity; the brighter the color, the larger is the size of its neighborhood. The thickness of a line denotes the intensity of a connection between a pair of given neurons. Thus a thick blue line indicates a strong positive connection, while a thin blue line also represents a positive connection but one with a lesser strength.

Figures 6,7 and 8 show that different age group activate different parts of the cube, as indicated by their connectivity.

Figures 6 shows that in age group (0-3), the Facial, Intercantal and Orbital width indexes have the strongest level of expression when compared to the two other age groups as they have stronger positive connections when compared to their counterparts in Figures 7 and 8. Changes in variables in a Spiking Neural Network result in increased spiking activity which in turn spawns positive connections. Thus we can conclude that Cranofacial growth in age group (0-3) occurs at a faster rate than with other two age groups.

Figure 7 shows that in age group (4-16) that there is strong connection strength in the Eye fissure and Mandibular index regions due to the presence of thick blue lines. Mandibular and Eye fissure indexes are associated with the eye and chin ratios. This suggests that most changes in the face occur in these features at age group (4-16).

Figure 8 shows that in age group (17-69) most of the strong connections seem to occur in the Orbital width, Intercantal, Mandibular and Facial index regions. Even though three of these features, namely the Facial, Intercantal and Orbital indexes, are also expressed in age goup (0-3), the levels of expression is less than that for age group (0-3). Thus we can conclude that while most changes in age group (17-69) occur in these indexes, the rate of change is slower with age goup (0-3).

Our comparative analysis shows that in all three age groups the Mandibular and Intercantal indexes are active as they are associated with strong spiking activity. The Eye fissure index creates strong spikes during age group (4-19) and (17-69). The Vermilion height index shows weak connections and a low level of spike activity in age groups (0-3) and (17-69). However, the Vermilion index shows a medium level of spike activity and strong connection weights in age grouping (4-16).

We observe that there is a clear shift away from the Facial, Intercantal, Orbital width and Mouth Face width indexes as markers for the age group (0-3) to Mandibular and Fissure indexes for age group (4-16). The fact that different indexes are prominent at different age groups suggests that NeuCube has succeeded in capturing the aging process. This is useful on two different accounts. First, it provides solid evidence that NeuCube is capturing not just the temporal but also spatial signals in the data. Second, from the viewpoint 


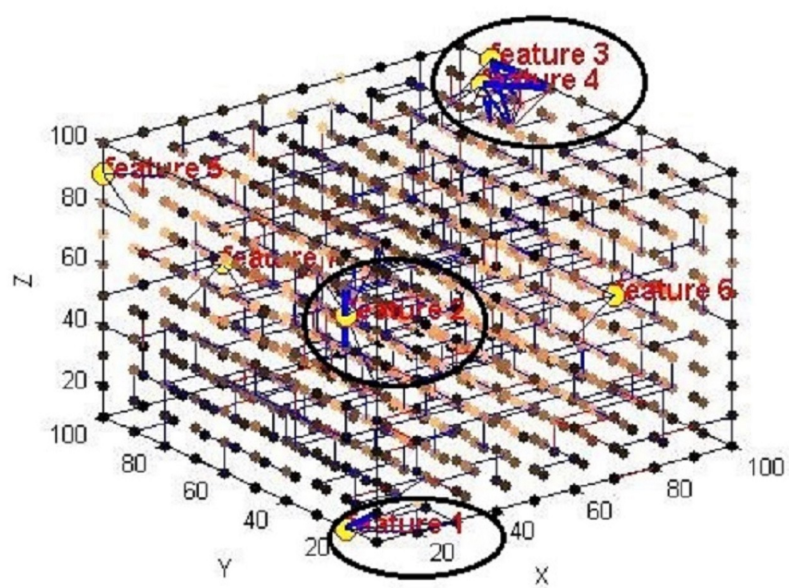

Key:

Feature 1 = Facial Index

Feature 2 = Mandibular index

Feature $3=$ Intercantal index

Feature 4 = Orbitabl width index

Feature $5=$ Eye fissure index

Feature 6= Vermilion height index

Feature 7= Mouth Face width index

Fig. 6 Age group (0-3) The size of the SNNc is controlled by three parameters: , $n_{x}, n_{y}$, and $z_{n}$, representing the neuron numbers along $\mathrm{x}, \mathrm{y}$ and $\mathrm{z}$ direction. And the total neuron number in the cube is $N=n_{x} \times n_{y} \times n_{z}$.A SNNc of 1000 (10 x 10 $\mathrm{x}$ 10) neurons are shown in above aging model.

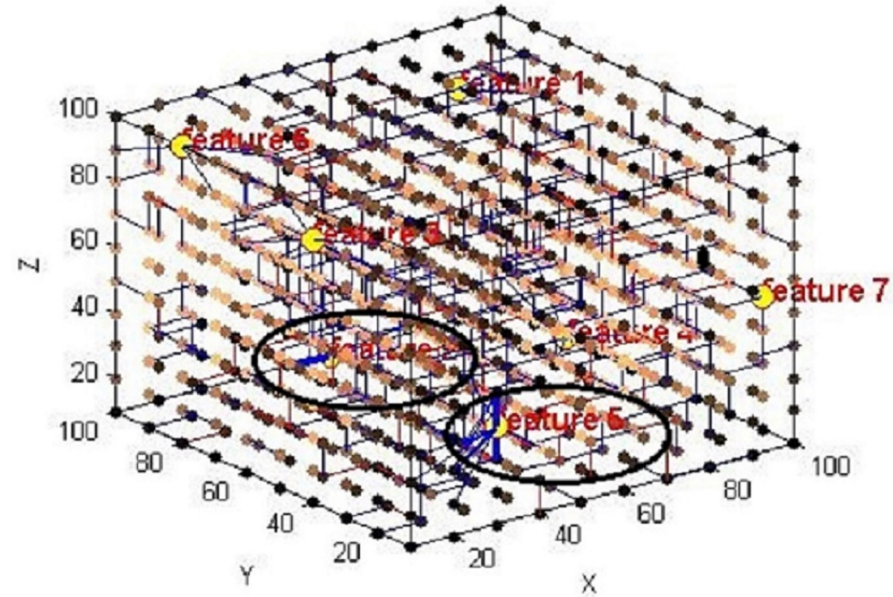

Key:

Feature 1 = Facial Index

Feature 2 = Mandibular index

Feature $3=$ Intercantal index

Feature 4 = Orbitabl width index

Feature $5=$ Eye fissure index

Feature $6=$ Vermilion height index

Feature $7=$ Mouth Face width index

Fig. 7 Age group (4-16)

of aging research it provides a useful insight into the changes that take place in the face over time and enables interested researchers into building more useful age estimation models. It also provides the potential to simulate future changes in a person's face over time.
Table 9 Feature profile for age group (17-69)

\begin{tabular}{|l|l|l|}
\hline \hline Features & $\begin{array}{l}\text { Influence } \\
\text { Level }\end{array}$ & Relationship \\
\hline Facial & Medium & Weak \\
Mandibular & High & Strong \\
Intercantal & Medium & Strong \\
Orbital width & High & Strong \\
Eye fissure & High & Weak \\
Vermilion height & Low & Weak \\
Mouth Face Width & Low & Weak \\
\hline
\end{tabular}




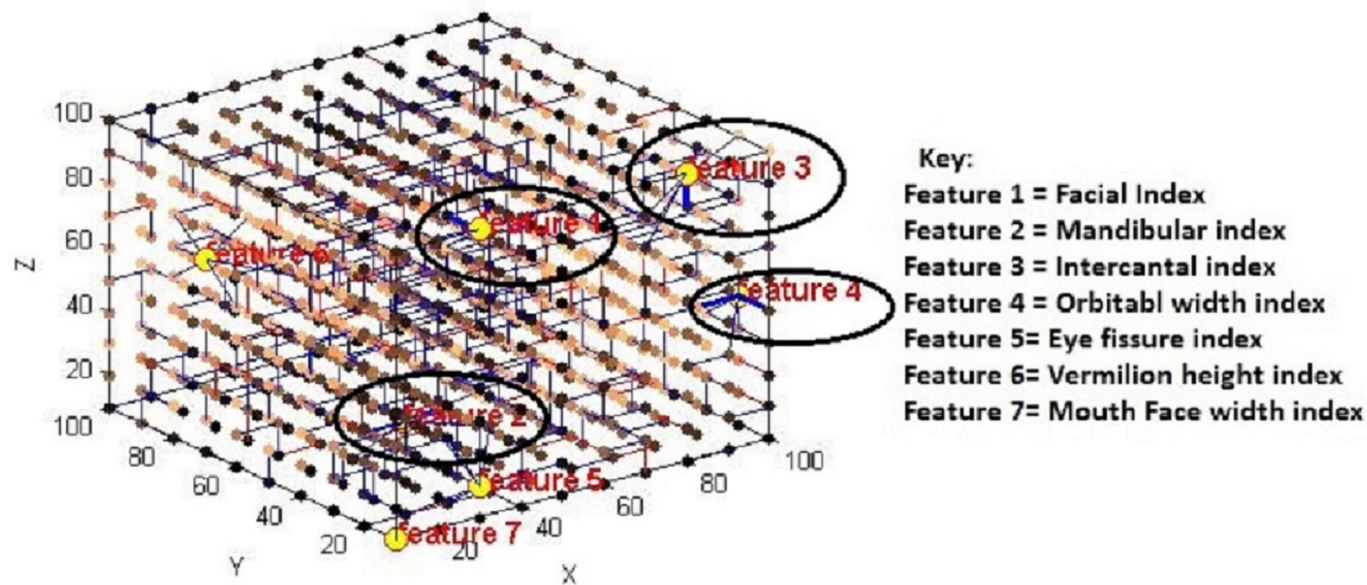

Fig. 8 Age group (17-69)

Table 7 Feature profile for age group (0-3)

\begin{tabular}{|l|l|l|}
\hline \hline Features & $\begin{array}{l}\text { Influence } \\
\text { Level }\end{array}$ & Connections \\
\hline Facial & High & Strong \\
Mandibular & High & Strong \\
Intercantal & High & Strong \\
Orbital width & High & Strong \\
Eye fissure & Low & Weak \\
Vermilion height & Low & low \\
Mouth Face Width & Medium & strong \\
\hline
\end{tabular}

Table 8 Feature profile for age group (4-16)

\begin{tabular}{|l|l|l|}
\hline \hline Features & $\begin{array}{l}\text { Influence } \\
\text { Level }\end{array}$ & Relationship \\
\hline Facial & Low & Weak \\
Mandibular & High & Strong \\
Intercantal & Medium & Weak \\
Orbital width & Low & Weak \\
Eye fissure & High & Strong \\
Vermilion height & Medium & Weak \\
Mouth Face Width & Low & Weak \\
\hline
\end{tabular}

We conclude this section by observing that NeuCube not only outperformed its rivals across both databases in terms of classification accuracy but also yielded useful insights into the aging process.

\section{Empirical Results of Gender Recognition}

\subsection{Gender Recognition with FG-NET}

For this experiment we use the FG-NET Database. We model Gender recognition as a two-class classification problem. We assign the gender label through a visual inspection of the data, with class 1 as male and class 2 as female. We also used the classical classifiers from Weka as in the first experiment for comparative analysis. For each of the images we take 7 features. Each sample provides a $5 \times 7$ matrix, ie 5 images, each having 7 features. In all there are 172 samples. The leave-oneperson-out testing strategy is used for FG-NET. Table 10 shows the results of the gender recognition classification process.

Table 10 Gender Classification on FG-NET

\begin{tabular}{lllll}
\hline Measure & NeuCube & MLP & KNN & NaiveBayes \\
\hline \hline Accuracy(\%) & 95.00 & 76.10 & 83.00 & 62.79 \\
\hline
\end{tabular}

Tables 10 and 11 show clearly that NeuCube has better classification performance when compared to all of its rivals. 
Table 11 Age group classification on FG-NET

\begin{tabular}{l|l|}
\hline \hline Models & Classification(\%) \\
\hline \hline NeuCube & $95 \%$ \\
(Guo and Mu 2011) & $87.20 \%$ \\
\hline \hline
\end{tabular}

5.2 Gender Recognition with FG-NET in a younger age group (0-18)

For this experiment we use the FG-NET Database. For each of the images we take 7 features. Each sample provides a $5 \times 7$ matrix ie 5 images, with each having 7 features. In all there are 120 samples. The leave-oneperson-out testing strategy was used for FG-NET. Table 12 shows results of gender recognition in the younger age group.

Table 12 Gender Classification for younger age group (0-18)

\begin{tabular}{lllll}
\hline Measure & NeuCube & MLP & KNN & NaiveBayes \\
\hline \hline Accuracy(\%) & 85.00 & 72.60 & 70.80 & 67.3 \\
\hline
\end{tabular}

\subsection{Gender Recognition with MORPH}

For this experiment we use the MORPH Database. We model Gender recognition as a two-class classification problem. We use the same testing strategy as used in (Guo and Mu 2011; Liu et al 2015). This data is then fed into the NeuCube. We use the same classical classifiers from Weka as we did in the first experiment for comparative analysis. For each of the images we take 7 features. Each sample provides a $5 \times 7$ matrix ie 5 images, with each having 7 features. In all there are 3378 samples.

Table 13 Results of Gender Classification using a NeuCube eSTDM in comparison with traditional techniques: Knn, MLP, NB

\begin{tabular}{lllll}
\hline Measure & NeuCube & MLP & KNN & NaiveBayes \\
\hline \hline Accuracy(\%) & 99.00 & 76.30 & 88.00 & 66.00 \\
\hline
\end{tabular}

Tables 13 and 14 show clearly that NeuCube has better classification performance when compared to all of its rivals.
Table 14 Comparative results of age group classification with FG-NET database

\begin{tabular}{l|l|}
\hline \hline Models & Classification(\%) \\
\hline \hline NeuCube & $99 \%$ \\
$($ Guo and Mu 2011) & $98.20 \%$ \\
\hline \hline
\end{tabular}

5.4 Gender Recognition with MORPH in a younger age group (16-20)

In all there are 420 samples that we use for this experiment. Each sample provides a 5x7 matrix with each image having 7 features. We use the same testing strategy as used in (Guo and Mu 2011; Liu et al 2015). Table 15 shows results of gender recognition in the younger age groups.

Table 15 Results of Gender Classification at a younger age group (16-20) using a NeuCube eSTDM in comparison with traditional techniques: KNN, MLP, NB

\begin{tabular}{lllll}
\hline Measure & NeuCube & MLP & KNN & NaiveBayes \\
\hline Accuracy $(\%)$ & 87.0 & 68.3 & 79.3 & 63.8 \\
\hline
\end{tabular}

It was observed that the gender recognition classification achieved with NeuCube was better than with other traditional techniques for the younger age groups.

Discussion In Figures 9 and 10, line colour, thickness and coloring of neurons have similar meaning to that described above.

By visualization of the cube in Figure 9 we can see that the Intercantal, Vermilion height, Eye fissure and Mouth Face Width indexes have strong positive connections. However, the Mandibular, Facial and orbital indexes show weak connections caused by less spiking activity.

In Figure 10 we see that all features generate high spikes and stronger connections except for the Vermilion height index. This is typical for the female specimens in the data sample.

By conducting a comparative analysis we observe that in both genders that the Intercantal, Eye Fissure and Mouth Face Width indexes show strong positive connections, implying that more changes would occur in eyes, mouth and bone structure between eyes.

The Facial, Mandibular, Orbital width and Vermilion height indexes behave differently across the genders, implying that a gender aging pattern exists. This further validates the point that gender also effects the face 


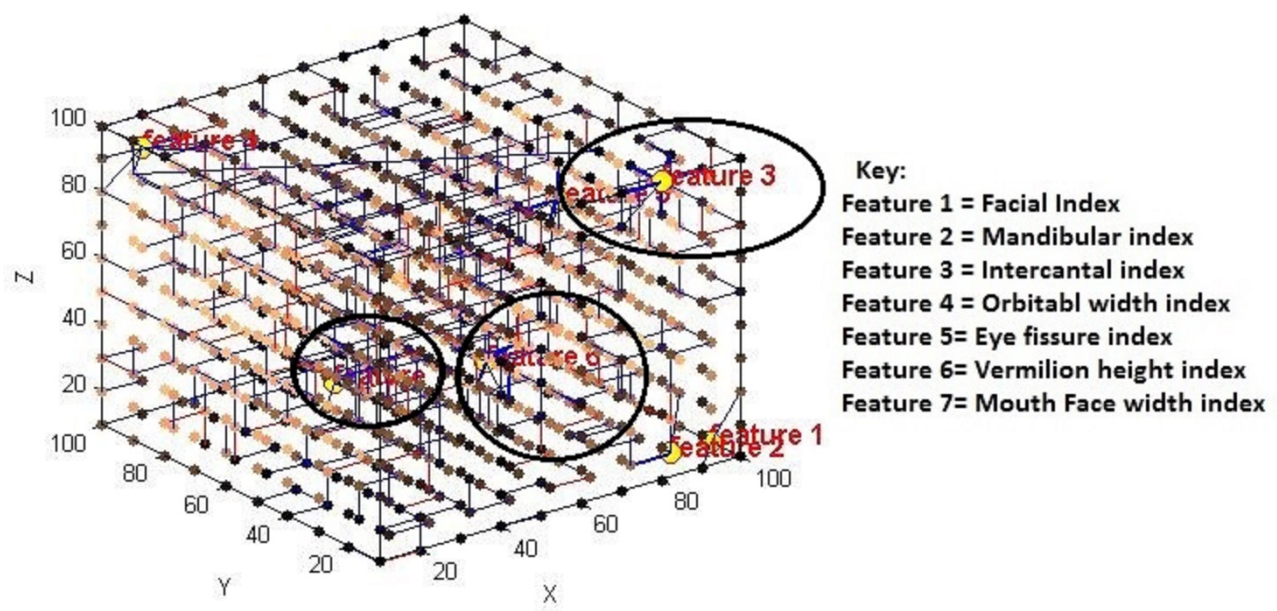

Fig. 9 Male

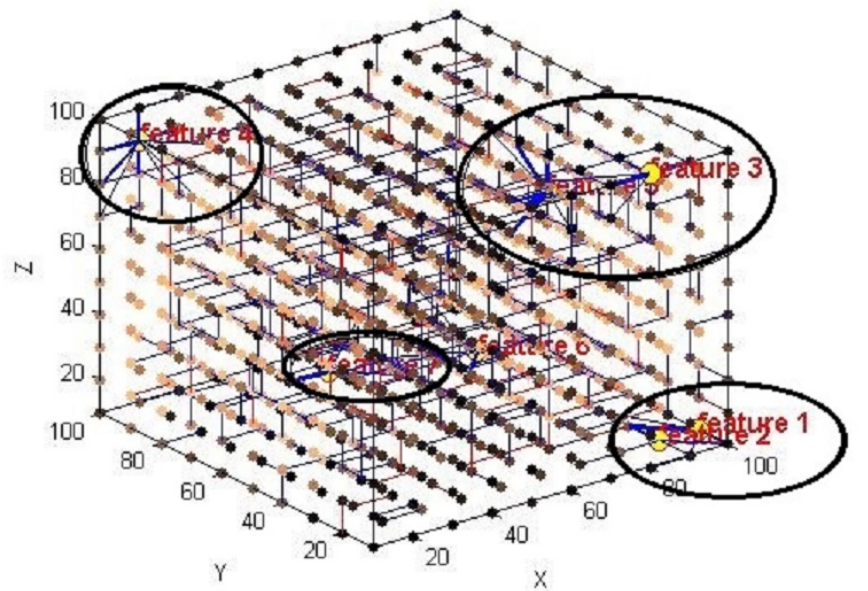

Key:

Feature 1 = Facial Index

Feature 2 = Mandibular index

Feature $3=$ Intercantal index

Feature $4=$ Orbitabl width index

Feature 5= Eye fissure index

Feature $6=$ Vermilion height index

Feature $7=$ Mouth Face width index

Fig. 10 Female

recognition process. Therefore, it is recommended that gender-specific aging models be built for age invariant face recognition.

By visualization we can also see another interesting effect, facial index, mandibular index, intercantal index and orbital index show strong connections for female gender as compared to male gender. It may be con- cluded that female gender ages more quickly as compared to male gender.

In these experiments we can see that the NeuCube has the capability of providing better accuracy as compared to the traditional methods. Its main strength lies in three dimensional connections and their weights and the ability of learning spatio temporal aging process. 
Table 16 Feature profile (Male)

\begin{tabular}{|l|l|l|}
\hline \hline Features & $\begin{array}{l}\text { Influence } \\
\text { Level }\end{array}$ & Connections \\
\hline Facial & Low & Weak \\
Mandibular & Low & Weak \\
Intercantal & High & Strong \\
Orbital width & Low & Weak \\
Eye fissure & High & Strong \\
Vermilion height & High & Strong \\
Mouth Face Width & High & strong \\
\hline
\end{tabular}

Table 17 Feature profile (Female)

\begin{tabular}{|l|l|l|}
\hline \hline Features & $\begin{array}{l}\text { Influence } \\
\text { Level }\end{array}$ & Connections \\
\hline Facial & High & Strong \\
Mandibular & High & Strong \\
Intercantal & High & Strong \\
Orbital width & High & Strong \\
Eye fissure & High & Strong \\
Vermilion height & Low & low \\
Mouth Face Width & High & strong \\
\hline
\end{tabular}

\section{Conclusion}

In this paper we researched two inter-related problems in face recognition using the NeuCube computational platform. The well-known FG-Net and Morph image galleries were used and Anthropometric features were extracted from landmark points on the face. These features enabled learning of spatio temporal relationships. The Weka machine learning workbench was used to compare the performance of traditional classifiers such as the K nearest neighbor (Knn), Multi-LayerPerceptron (MLP) and Naive Bayes with NeuCube. This research has revealed that NeuCube has the capability of giving better performance for gender recognition and age group classification because it has the capacity to learn both spatial and temporal relationships simultaneously.

Our empirical results show that NeuCube performed consistently better across both problem types that we investigated. We explored the temporal relationships between different Anthropometric features. The aging process causes significant alterations on the human face, thus affecting the long term performance of face authentication systems. Our research also revealed that different Fiducial markers are prominent at different age groups, thus offering the possibility of estimating aging effects for future points in a given person's timeline. However, for age simulation to be successful such markers may need to be combined with other personspecific information such as lifestyle and environment variables that could also impact on shaping a person's facial profile over time.

One direction that can be explored is to construct NeuCube aging models for each feature separately and then fuse the different models to build a composite model of the simulated face. Modelling each feature separately is attractive as it allows each feature to express its individual contribution to the aging process to the maximum possible extent without interference from the other features. Our future research will explore this approach in depth.

In conclusion we observe that NeuCube provides a good platform for aging research, whether the objective is age estimation or estimating change in facial features over time, thus opening up future research opportunities in these two key areas of aging research.

\section{References}

Alvi FB, Pears R (2015a) An integrated modeling approach to age invariant face recognition. In: Sixth International Conference on Graphic and Image Processing (ICGIP 2014), International Society for Optics and Photonics, pp 94,430A-94,430A

Alvi FB, Pears R (2015b) Texture modelling for age invariant face recognition. In: Proceedings of the International Conference on Image Processing, Computer Vision, and Pattern Recognition (IPCV), The Steering Committee of The World Congress in Computer Science, Computer Engineering and Applied Computing (WorldComp), p 24

Alvi FB, Pears R (2015c) Use of spatio-temporal modeling for age invariant face recognition. In: Proceedings of 30th International Conference on Image and Vision Computing New Zealand (IVCNZ 2015), IEEE

Alvi FB, Pears R (2016) A composite spatio-temporal modeling approach for age invariant face recognition. Expert Systems with Applications

Biswas S, Aggarwal G, Ramanathan N, Chellappa R (2008) A non-generative approach for face recognition across aging. In: Biometrics: Theory, Applications and Systems, 2008. BTAS 2008. 2nd IEEE International Conference on, IEEE, pp 1-6

Cootes TF, Edwards GJ, Taylor CJ, et al (1999) Comparing active shape models with active appearance models. In: BMVC, vol 99, pp 173-182

Dhoble K, Nuntalid N, Indiveri G, Kasabov N (2012) Online spatio-temporal pattern recognition with evolving spiking neural networks utilising address event representation, rank order, and temporal spike learning. In: Neural Networks (IJCNN), The 2012 International Joint Conference on, IEEE, pp 1-7

Farkas LG (1994) Anthropometry of the Head and Face. Raven Pr

Farkas LG, Munro IR (1987) Anthropometric facial proportions in medicine. Charles C. Thomas Publisher

FG-NET (2002) Fg-net database. URL http://www-prima. inrialpes.fr/FGnet/

Fu Y, Huang TS (2008) Human age estimation with regression on discriminative aging manifold. Multimedia, IEEE Transactions on 10(4):578-584 
Geng X, Zhou ZH, Smith-Miles K (2007) Automatic age estimation based on facial aging patterns. IEEE Transactions on Pattern Analysis and Machine Intelligence 29(12):2234-2240

Gunay A, Nabiyev VV (2008) Automatic age classification with lbp. In: Computer and Information Sciences, 2008. ISCIS'08. 23rd International Symposium on, IEEE, pp $1-4$

Guo G, Mu G (2011) Simultaneous dimensionality reduction and human age estimation via kernel partial least squares regression. Proceedings of the IEEE Computer Society Conference on Computer Vision and Pattern Recognition pp 657-664, DOI 10.1109/CVPR.2011.5995404

Guo G, Fu Y, Dyer CR, Huang TS (2008) Image-based human age estimation by manifold learning and locally adjusted robust regression. Image Processing, IEEE Transactions on $17(7): 1178-1188$

Hechenbichler K, Schliep K (2004) Weighted k-nearestneighbor techniques and ordinal classification. Tech. rep., Discussion paper//Sonderforschungsbereich 386 der Ludwig-Maximilians-Universität München

Horng WB, Lee CP, Chen CW (2001) Classification of age groups based on facial features. 4(3):183-192

Jain AK, Nandakumar K, Ross A (2016) 50 years of biometric research: Accomplishments, challenges, and opportunities. Pattern Recognition Letters

Kasabov N (2007) Evolving connectionist systems: the knowledge engineering approach. Springer Science \& Business Media

Kasabov N (2012) Neucube evospike architecture for spatiotemporal modelling and pattern recognition of brain signals. In: Artificial Neural Networks in Pattern Recognition, Springer, pp 225-243

Kasabov N, Dhoble K, Nuntalid N, Indiveri G (2013) Dynamic evolving spiking neural networks for on-line spatioand spectro-temporal pattern recognition. Neural Networks 41:188-201

Kasabov N, Feigin V, Hou ZG, Chen Y, Liang L, Krishnamurthi R, Othman M, Parmar P (2014) Evolving spiking neural networks for personalised modelling, classification and prediction of spatio-temporal patterns with a case study on stroke. Neurocomputing 134:269-279

Kasabov N, Scott NM, Tu E, Marks S, Sengupta N, Capecci E, Othman M, Doborjeh MG, Murli N, Hartono R, et al (2015) Evolving spatio-temporal data machines based on the neucube neuromorphic framework: Design methodology and selected applications. Neural Networks

Kasabov N, Scott NM, Tu E, Marks S, Sengupta N, Capecci E, Othman M, Doborjeh MG, Murli N, Hartono R, et al (2016) Evolving spatio-temporal data machines based on the neucube neuromorphic framework: design methodology and selected applications. Neural Networks 78:1-14

Kasabov NK (2014) Neucube: A spiking neural network architecture for mapping, learning and understanding of spatio-temporal brain data. Neural Networks 52:62-76

Kwon YH, da Vitoria Lobo N (1999) Age classification from facial images. Computer Vision and Image Understanding 74(1):1-21

Lanitis A, Taylor CJ, Cootes TF (1999) Modeling the process of ageing in face images. In: The Proceedings of the Seventh IEEE International Conference on Computer Vision, IEEE, vol 1, pp 131-136

Lanitis A, Draganova C, Christodoulou C (2004) Comparing different classifiers for automatic age estimation. IEEE Transactions on Systems, Man, and Cybernetics, Part B (Cybernetics) 34(1):621-628
Levi G, Hassner T (2015) Age and gender classification using convolutional neural networks. In: Proceedings of the IEEE Conference on Computer Vision and Pattern Recognition Workshops, pp 34-42

Li Z, Park U, Jain AK (2011) A discriminative model for age invariant face recognition. Information Forensics and Security, IEEE Transactions on 6(3):1028-1037

Liu KH, Yan S, Kuo CCJ (2015) Age Estimation via Grouping and Decision Fusion. IEEE Transactions on Information Forensics and Security 10(11):2408-2423, DOI 10.1109/TIFS.2015.2462732, URL http://ieeexplore.ieee.org/lpdocs/epic03/ wrapper.htm?arnumber $=7173035$

Milborrow S, Nicolls F (2014) Active shape models with sift descriptors and mars. In: VISAPP (2), pp 380-387

Ramanathan N, Chellappa R (2006) Modeling age progression in young faces. In: Computer Vision and Pattern Recognition, 2006 IEEE Computer Society Conference on, IEEE, vol 1, pp 387-394

Ricanek Jr K, Tesafaye T (2006) Morph: A longitudinal image database of normal adult age-progression. In: Automatic Face and Gesture Recognition, 2006. FGR 2006. 7th International Conference on, IEEE, pp 341-345

Sai PK, Wang JG, Teoh EK (2015) Facial age range estimation with extreme learning machines. Neurocomputing 149(Part A):364-372, DOI 10.1016/j.neucom.2014.03. 074, URL http://dx.doi.org/10.1016/j.neucom. 2014. 03.074

Schliebs S, Kasabov N (2013) Evolving spiking neural networka survey. Evolving Systems 4(2):87-98

Schliebs S, Fiasché M, Kasabov N (2012) Constructing robust liquid state machines to process highly variable data streams. In: International Conference on Artificial Neural Networks, Springer, pp 604-611

Sengupta N, Scott N, Kasabov N (2015) Framework for knowledge driven optimisation based data encoding for brain data modelling using spiking neural network architecture. In: Proceedings of the Fifth International Conference on Fuzzy and Neuro Computing (FANCCO-2015), Springer, pp 109-118

Song S, Miller KD, Abbott LF (2000) Competitive hebbian learning through spike-timing-dependent synaptic plasticity. Nature neuroscience 3(9):919-926

Thukral P, Mitra K, Chellappa R (2012) A hierarchical approach for human age estimation. In: 2012 IEEE International Conference on Acoustics, Speech and Signal Processing (ICASSP), IEEE, pp 1529-1532

Tu E, Kasabov N, Othman M, Li Y, Worner S, Yang J, Jia Z (2014) Neucube (st) for spatio-temporal data predictive modelling with a case study on ecological data. In: 2014 International Joint Conference on Neural Networks (IJCNN), IEEE, pp 638-645

Wang JG, Yau WY, Wang HL (2009) Age categorization via ecoc with fused gabor and lbp features. In: Applications of Computer Vision (WACV), 2009 Workshop on, IEEE, pp 1-6

Wang JG, Sung E, Yau WY (2011) Active learning for solving the incomplete data problem in facial age classification by the furthest nearest-neighbor criterion. IEEE transactions on image processing 20(7):2049-2062

Yan S, Zhou X, Liu M, Hasegawa-Johnson M, Huang TS (2008) Regression from patch-kernel. In: Computer Vision and Pattern Recognition, 2008. CVPR 2008. IEEE Conference on, IEEE, pp 1-8 